\title{
Las aguas subterráneas en el Arco Seco de Panamá: Un tesoro por explorar
}

\author{
Castrellón, María G. \\ Universidad Tecnológica de Panamá, Panamá \\ Panamá, Panamá \\ mariag.castrellon@gmail.com \\ Muñoz, Melisabel \\ Universidad Tecnológica de Panamá, Panamá \\ Panamá, Panamá \\ melisabel.munoz@utp.ac.pa \\ Guerra-Chanis, Gisselle \\ Universidad Tecnológica de Panamá \\ Ciudad de Panamá, Panamá \\ gisselle.guerra@utp.ac.pa \\ Leiva, Jorge A. \\ Universidad de Costa Rica \\ Liberia, Costa Rica \\ jorge.leivasanabria@ucr.ac.cr \\ Foglia, Laura \\ University of California, Davis \\ Davis, U.S.A. \\ Ifoglia@ucdavis.edu \\ Fábrega, José \\ Universidad Tecnológica de Panamá, Panamá \\ Panamá, Panamá \\ jose.fabrega@utp.ac.pa
}

\section{ABSTRACT}

The Dry Arc of Panama is located within the provinces of Herrera, Los Santos and Coclé. It has the lowest precipitation of the country, with $400 \mathrm{~mm}$ (dry season) and $1600 \mathrm{~mm}$ (wet season). Deforestation and excessive well construction affect the soil and the availability 
of groundwater. Hydrogeology and pedology studies are the foundation for land-use plans and water resources management plans. Two projects are underway in this region, one in the Estibaná river sub-catchment (SRE) and another in the Zaratí river sub-catchment (SRZ), located in Los Santos and Coclé, respectively. Geological data, including maps and borehole descriptions, were used to identify the main hydrogeological units in both study areas. The interaction between surface and groundwater was evaluated from river discharge and piezometric levels. In both SRE and SRZ the piezometric level is shallow ( $\sim 10 \mathrm{~m})$ and rivers are gaining water from the aquifer. Soils in SRZ were classified using drill hole techniques in 136 points. Ultisoles soils in the upper portion have a low hydraulic conductivity, while in the middle portion older-Ultisoles have low infiltration. The upper portion has Entisoles and Inceptisoles with high organic content.

Keywords: Dry Arc, groundwater resources, water sustainability, USDA soil taxonomy, soil properties.

\section{RESUMEN}

El Arco Seco de Panamá está ubicado en las provincias de Herrera, Los Santos y Coclé. Tiene la precipitación anual más baja del país, con un máximo de $400 \mathrm{~mm}$ (temporada seca) y $1600 \mathrm{~mm}$ (temporada lluviosa). La deforestación y la construcción descontrolada de pozos afecta el suelo y la disponibilidad de aguas subterráneas. Investigaciones hidrogeológicas y pedológicas son la base para el desarrollo de planes de uso de suelo y de manejo del recurso hídrico. Dos proyectos se llevan a cabo en esta región, uno en la subcuenca del río Estibaná (SRE), y otro en la subcuenca del río Zaratí (SRZ), en Los Santos y Coclé, respectivamente. Datos geológicos, incluyendo mapas y registros de perforación se utilizaron para identificar las principales unidades hidrogeológicas del área de estudio. La interacción entre aguas superficiales y subterráneas se evaluó a partir de mediciones de caudales en ríos y niveles piezométricos en pozos. Los resultados indican que el nivel piezométrico es somero ( 10 $\mathrm{m}$ ) para ambas subcuencas y los ríos ganan agua del acuífero. Los suelos de la subcuenca del río Zaratí se clasificaron usando técnicas de prospección de barreno en 136 puntos. Los suelos Ultisoles de la parte alta tienen una conductividad hidráulica lenta mientras que en la parte media son más antiguos con una baja infiltración. Los suelos Entisoles e Inceptisoles, con alto contenido orgánico, predominan en la cuenca alta.

Palabras claves: Arco Seco, recursos de agua subterránea, sostenibilidad hídrica, taxonomía de suelos USDA, propiedades de suelos. 


\section{INTRODUCCIÓN}

El Arco Seco de Panamá se localiza en las provincias de Herrera, Los Santos y Coclé. Es la región con el rango de precipitaciones más bajas del país ( 0 a $400 \mathrm{~mm}$ durante la estación seca y 550 a 1600 mm durante la estación lluviosa) y es propensa a periodos de sequía prolongados, que pueden extenderse hasta siete meses en años que coinciden con el Fenómeno de El Niño. El estrés hídrico es notorio en el paisaje del Arco Seco y se acentúa por las malas prácticas de uso de suelo. La deforestación en zonas montañosas es el principal problema ambiental para garantizar la recarga de las reservas de aguas subterráneas. Por otro lado, la construcción de pozos en la zona se ha dado de una manera desordenada. En general, la información hidrogeológica es muy limitada y esto dificulta el estudio de aguas subterráneas en la región.

El objetivo del simposio "Las aguas subterráneas en el Arco Seco de Panamá: Un tesoro por explorar" es divulgar el estado actual de la hidrogeología del Arco Seco y los resultados de investigaciones recientes, y discutir futuras líneas de investigación en elárea. Se contó con la participación de investigadores de Costa Rica, Estados Unidos y Panamá. En el simposio se expusieron temas sobre la situación actual del recurso hídrico en el Arco Seco, el desarrollo de modelos hidrogeológicos conceptuales, la importancia de la interacción entre aguas superficiales y subterráneas para garantizar la sostenibilidad del recurso hídrico, y la génesis, caracterización e importancia de los suelos para la hidrología. Al finalizar contamos con la participación de todos los panelistas y moderador para la discusión final, conclusiones y lecciones aprendidas. El simposio resalta los avances de los proyectos IOMA 17-006 llevado a cabo en la subcuenca río Estibaná (SRE), en la provincia de Los Santos y el FID 17-084 en la subcuenca del río Zaratí (SRZ), en la provincia de Coclé. Ambos proyectos son financiados por la Secretaría Nacional de Ciencia, Tecnología e Innovación de Panamá (SENACYT).

\section{METOdOLOGÍA}

\section{A. Contexto geológico y unidades hidrogeológicas}

Un modelo hidrogeológico conceptual es una descripción cualitativa del comportamiento de las aguas subterráneas de una región, en dónde se integra la información existente sobre la geología, estratigrafía, hidrología, y el clima de una zona en específico. Esta descripción o caracterización se considera como una simplificación del comportamiento hidrogeológico de una región, pero que describe en suficiente detalle los procesos más importantes necesarios para la abordar los objetivos del ejercicio de modelación [1].

La construcción del modelo hidrogeológico conceptual en SER y SRZ inició con una revisión extensa de mapas y descripciones geológicas existentes, registros de perforación del IDAAN y datos de pruebas de bombeo. Esta información se utilizó para identificar las principales unidades hidrogeológicas en cada una de estas regiones. Una unidad hidrogeológica 
es un conjunto de formaciones geológicas que aunque cuentan con diferentes tipos de roca, tienen similar comportamiento hidráulico (p. ej., similar porosidad o conductividad hidráulica).

\section{B. Interacción de Aguas Superficiales y Subterráneas}

El intercambio entre aguas superficiales y subterráneas es fundamental en el ciclo hidrológico. Dependiendo del clima de una región, las aguas superficiales suministran recarga al acuífero subyacente, o el acuífero descarga y nutre a los cuerpos de aguas superficiales. La representación conceptual de la interacción de aguas superficiales y subterráneas utilizando los datos existentes es clave para entender el comportamiento hidrológico de una región. En SRE y SRZ esta interacción se analizó a partir de mediciones de caudal realizadas a lo largo de los ríos Estibaná y Zaratí, respectivamente, durante la temporada seca. La diferencia en el caudal base medido entre dos puntos en dichos ríos se utilizó para determinar si los ríos pierden o ganan agua del acuífero.

Adicionalmente, se estableció una red de monitoreo de niveles piezométricos en ambas subcuencas a partir de pozos de producción inactivos (de 15 a $20 \mathrm{~cm}$ de diámetro y de 30 a $100 \mathrm{~m}$ de profundidad) en su mayoría del IDAAN. Se identificaron un total de 16 y 19 pozos de monitoreo en Estibaná y Zaratí, respectivamente. La profundidad al nivel estático se midió mensualmente en dichos pozos y estos datos se procesaron para generar mapas de elevación de la superficie piezométrica utilizando técnicas de interpolación. La dirección principal de flujo de aguas subterráneas se identificó a partir de dichos mapas dibujando líneas de corriente perpendiculares a las cotas de igual nivel piezométrico o isopiezas.

\section{Clasificación taxonómica, caracterización del suelo e importancia hidrológica}

El suelo mineral es un recurso natural crucial para la consecución de los objetivos de desarrollo sostenible de la ONU, porque su manejo y conservación está relacionado no solo a la seguridad alimentaria, sino al manejo sostenible en cuencas hidrográficas y la protección del recurso hídrico [2]. El estudio detallado de los suelos, su origen y características está en función de su distribución en el paisaje, clima, organismos, material parental (roca) y tiempo [3]. Consecuentemente, se consultaron mapas geológicos, de relieve, clima y uso de la tierra de la subcuenca del Río Zaratí (SRZ) para iniciar la delimitación de unidades cartográficas de suelos. Posteriormente, se realizó un trabajo de mapeo georeferenciado (escala 1:100 000), usando técnicas tradicionales de prospección de suelos en campo con barreno holandés y microcalicatas, donde se analizó la textura, color, profundidad, formaciones especiales, distribución de poros y raíces en los horizontes o capas de suelo. En total, se describieron 136 puntos de barreno a lo largo de la SRZ, distribuidos en 3 secciones de altitud: baja (17-102 m), media (96-342 m) y alta (231-685 m). Se identificaron 
14 perfiles o suelos modales (dominantes) y se levantaron calicatas (fosas) para describir y muestrear los horizontes maestros $(A, B, C)$ para análisis físicos y químicos. Los suelos se clasificaron según la Taxonomía de Suelos del USDA [4] y se evaluó su uso de la tierra actual y su importancia para la protección del recurso hídrico.

\section{RESULTADOS}

\section{A. Contexto geológico y unidades hidrogeológicas}

El sur del Arco Seco, correspondiente a la península de Azuero, es un área tectónicamente muy compleja, con presencia de fallas regionales en dirección Este-Oeste (Falla Ocú Parita) y Noroeste-Sureste (Falla Soná-Azuero). Predominan las rocas plutónicas, volcánicas y volcaniclástica del Cretácico Superior y el Eoceno, además de rocas sedimentarias consolidadas del Mioceno y Oligoceno [5]. Los acuíferos presentes en esta región son de porosidad secundaria, extensión local y de baja a media productividad. En la subcuenca del río Estibaná, localizada en la península de Azuero, se identificaron seis (6) diferentes unidades hidrogeológicas basadas en las diferentes formaciones geológicas existentes en la zona de estudio: Playa Venado, Quema, Valleriquito, Pesé, Santiago, y Changuinola-Ocú [6].

La parte noreste del Arco Seco, ubicada en las provincias de Coclé y Panamá Oeste, es de origen geológico más reciente que la península de Azuero. Las rocas volcánicas existentes en las zonas montañosas se originaron por la actividad volcánica asociada a la Formación Tucué y EI Valle del Mioceno Superior y Plioceno, respectivamente [7]. En el área de llanuras predominan las rocas sedimentarias de la Formación Río Hato del Cuaternario. La subcuenca del río Zaratí es geomorfológicamente un valle intermontano que se abre hacia una llanura aluvial. Las formaciones volcánicas del Terciario (El Valle y Cerro El Encanto) conforman el basamento de dicho valle y constituyen una unidad hidrogeológica de baja conductividad hidráulica (acuitardo). Los sedimentos consolidados y no consolidados de la Formación Río Hato que rellenan el valle y la llanura aluvial conforman una unidad hidrogeológica de porosidad primaria de alta conductividad hidráulica. Esta unidad tiene gran potencial para el almacenamiento y explotación de aguas subterráneas y constituye uno de los acuíferos más importantes de la región.

\section{B. Interacción de Aguas Superficiales y Subterráneas}

El caudal base de los ríos Estibaná y Zaratí aumentan a lo largo de su trayecto debido al aporte de aguas subterráneas del acuífero. Esta dinámica es característica de llanuras húmedas e indica que los ríos son puntos de descarga de aguas subterráneas. En SRE y SRZ, la profundidad al nivel piezométrico es relativamente somera (en su mayoría, en nivel en los pozos medidos no supera los $10 \mathrm{~m}$ ) y el nivel freático tiende a seguir el contorno de la 
topografía del terreno. El flujo de aguas subterráneas se da desde las partes altas de las cuencas hidrográficas hasta las partes bajas y descarga en los ríos.

\section{Clasificación taxonómica, caracterización del suelo e importancia hidrológica}

En la SRZ, los suelos de la parte baja son muy meteorizados, arcillosos, con conductividad hidráulica muy lenta en la superficie $(<0.01 \mathrm{~cm} \mathrm{~h}-1)$ debido a la presencia natural de ignimbritas (tobas) de origen ígneo (0.8-1.0 m prof.). Esto causa una tabla de agua somera que impide la infiltración a capas más profundas, por lo que los suelos son muy susceptibles a erosión hídrica por escorrentía superficial.

En la parte media, son suelos incluso más antiguos, pobres en nutrientes, con contenidos bajos de materia orgánica y de $\mathrm{pH}$ ácido, que los hace de difícil manejo para la producción agropecuaria y forestal. La presencia de plintita es dominante en el perfil (0.5-1.5 m prof.), la cual, es una capa arcillosa, masiva y con muy pobre infiltración, lo cual, limita su contribución a la recarga acuífera. En la parte alta, predominan suelos de textura francoarcillo-arenosa, con los mayores contenidos de materia orgánica. Estos suelos tienen un potencial agropecuario y forestal mayor. Por último, en la naciente del río Zaratí, presentó suelos recientes de texturas franco arenosas, las cuales se caracterizan por conductividades hidráulicas altas (14 a $33 \mathrm{~cm} \mathrm{h-1).} \mathrm{Es} \mathrm{decir,} \mathrm{estos} \mathrm{suelos} \mathrm{deben} \mathrm{mantenerse} \mathrm{bajo} \mathrm{cobertura}$ forestal permanente, ya sea bosque natural, bosques plantados o cultivos perennes (café bajo sombra de leguminosas, por ej.), promover un uso recreacional de ecoturismo, para así contribuir a la protección del suelo, fomentar la infiltración y la recarga de mantos acuíferos.

\section{CONCLUSIONES}

El Arco Seco es una región relativamente homogénea en cuanto a la precipitación, pero geológicamente muy heterogénea. Debido a los diferentes ambientes geológicos, en el Arco Seco se identifican dos regiones hidrogeológicas diferenciadas: 1) La península de Azuero con predominancia de roca volcánica fracturada y acuíferos locales de porosidad secundaria y 2) las llanuras de Coclé y Panamá Oeste en dónde los depósitos de sedimentos no consolidados conforman acuíferos de porosidad primaria. Esta última región tiene un enorme potencial hidrogeológico el cual todavía no ha sido explorado y por lo tanto necesitan más estudios especializados para determinar la extensión y productividad de los acuíferos de esta zona.

Los suelos de la parte baja y media de la subcuenca del Río Zaratí clasificaron como Ultisoles meteorizados, arcillosos, pobres en materia orgánica, con pobre infiltración, ricos en plintita, por lo que deben promoverse su recuperación por medio de coberturas forestales y enmiendas orgánicas. Los suelos de la parte alta clasificaron como Inceptisoles y Entisoles, de texturas más gruesas, mejor estructura y contenido de materia orgánica. Estos 
últimos deben ser protegidos para recarga acuífera mediante técnicas de conservación de suelos, reforestación y uso recreativo para ecoturismo.

La región del Arco Seco es un área con gran demanda del recurso hídrico y que durante gran parte del año presenta déficit. Es una región con gran potencial hídrico pero que requiere de estudios más profundos y el desarrollo e implementación de proyectos de monitoreo y control para poder implementar planes de gestión que puedan garantizar la sostenibilidad del recurso hídrico.

\section{REFERENCIAS}

[1] M. P. Anderson, W. W. Woessner, and R. J. Hunt, Applied Groundwater Modeling: Simulation of Flow and Advective Transport, Second. London, United Kingdom: Academic Press, 2015.

[2] S. D. Keesstra et al., "The significance of soils and soil science towards realization of the United Nations Sustainable Development Goals," SOIL, vol. 2, no. 2, pp. 111-128, 2016.

[3] S. W. Buol, R. J. Southard, R. C. Graham, and P. A. McDaniel, Soil Genesis and Classification. John Wiley \& Sons, Inc., 2011.

[4] Soil Survey Staff, Keys to Soil Taxonomy, 12 ed. Washington, DC: USDA-NRCS, 2014, p. 360.

[5] M. G. Castrellon Romero, "Characterization and Modeling of a Tropical Groundwater Basin: La Villa Watershed, Panama," University of California, Davis, 2018.

[6] M. G. Castrellon Romero, J. R. Fabrega, L. Foglia, A. Mojica, A. Ruiz, and S. Saavedra, "Groundwater Model of A Fractured Rock System As A Tool For Groundwater Management: The Estibaná SubCatchment, Azuero Peninsula, Panama," in The Conference Proceedings of the 38th IAHR Congress "Water Connecting the World," 2019, doi: https://doi.org/10.3850/38WC092019-1794.

[7] P. Salinas Serrano, "Modelo Conceptual Hidrogeológico de la cuenca del río Zaratí," Universidad Nacional de La Pampa, Argentina, 2010.

\section{AUTORIZACIÓN Y LICENCIA CC}

Los autores autorizan a APANAC XVIII a publicar el artículo en las actas de la conferencia en Acceso Abierto (Open Access) en diversos formatos digitales (PDF, HTML, EPUB) e integrarlos en diversas plataformas online como repositorios y bases de datos bajo la licencia CC: Attribution-NonCommercial-ShareAlike 4.0 International (CC BY-NC-SA 4.0) https://creativecommons. org/licenses/by-nc-sa/4.0/.

Ni APANAC XVIII ni los editores son responsables ni del contenido ni de las implicaciones de lo expresado en el artículo. 